\title{
Influencia de la soldabilidad de un acero inoxidable austenitico
}

\author{
Influence of the safety of a sustainable stainless steel
}

\section{Influência da segurança de um aço inoxidável sustentável}

\author{
Ernesto Camargo-Suarez ${ }^{1}$ \\ Leonardo Bohórquez-Espinosa² \\ Mónica Katherine Sánchez-Alarcón ${ }^{3}$
}

Recibido: mayo de 2018

Aceptado: agosto de 2018

Para citar este artículo: Camargo-Suarez, E., Bohórquez-Espinosa, L., y Sánchez-Alarcón, M.K. (2018). Influencia de la soldabilidad de un acero inoxidable austenitico. Revista Científica, 33(3), 275-286. Doi: https://doi. org/10.14483/23448350.13762

\section{Resumen}

La empresa Masa Stork, reconocida en el sector de hidrocarburos en Colombia, se vio en la necesidad de utilizar en un proyecto de generación de energía tubería de 2 pulgadas para transporte de aguas residuales en la refrigeración de moto-generadoras pero posteriormente se presentó un problema de fractura en la soldadura cuando estas se pusieron en funcionamiento. Cuando los aceros inoxidables son calentados en un rango de temperatura de $500^{\circ} \mathrm{C}$ a $700^{\circ} \mathrm{C}$, el carbono que contiene el acero reacciona con el cromo y forma carburos de cromo que no tienen las mismas características del cromo aleado. Estas reacciones empobrecen el acero y hacen que disminuyan sus propiedades en la zona calentada. Esta investigación se enfocó en la determinación de la influencia de la temperatura en la Zona Afectada Termodinámicamente (ZAT) de una soldadura en acero inoxidable AISI 316, bajo proceso GTAW, soldado con electrodo ER316 al ser sometido a temperatura de precalentamiento antes de la soldadura y su comportamiento ante tratamiento térmico. Se determinó que en el soldeo debe tenerse en cuenta una baja aportación de calor, distribuyendo el calor en forma equilibrada para que este sea repartido en la pieza de la forma más simétricamente posible. Además, se debe procurar que el nivel de embriamiento sea lo más bajo posible, así se consigue que la ZAT sea más estrecha.

Palabras clave: soldabilidad, aceros inoxidables austeníticos, precalentamiento.

\begin{abstract}
The Masa Stork Company recognized in the hydrocarbons sector in Colombia, in a power generation project saw the need to use 2-inch tubing to transport wastewater in the cooling of motor-generators, presenting the problem of fracture in the welding when they are put into operation. When stainless steels are heated in a temperature range of $500{ }^{\circ} \mathrm{C}$ to $700{ }^{\circ} \mathrm{C}$, the carbon- containing the steel reacts with the chromium to form chromium carbides that
\end{abstract}

Universidad Pedagógica y Tecnológica de Colombia. Boyacá, Colombia. ernesto.camargo@uptc.edu.co

Universidad Pedagógica y Tecnológica de Colombia. Boyacá, Colombia. ernes.camar@uptc.edu.co

Universidad Pedagógica y Tecnológica de Colombia. Boyacá, Colombia. moni.sanchez@uptc.edu.co 
do not have the same characteristics as the alloyed chromium. These reactions deplete the steel and reduce its properties in the heated zone. This investigation focused in the determination of the influence of the temperature in the ZAT of a welding in stainless steel AISI 316, under GTAW process, welded with ER316 electrode when subjected to pre-heating temperature before welding and its behavior before heat treatment; and determining that in the welding a low contribution of heat must be taken into account, distributing the heat in a balanced way so that it is distributed in the piece as symmetrically as possible. In addition, it must be ensured that the level of embrittlement is as low as possible, thus making the Zone Affected by heat more narrow.

Keywords: weldability, austenitic stainless steels, pre-heating.

\section{Resumo}

A empresa Masa Stork, reconhecida no setor de hidrocarbonetos na Colômbia, em um projeto de geração de energia, viu a necessidade de usar tubos de 2 polegadas para transportar águas residuais no resfriamento de motores-geradores, apresentando o problema de fratura em a soldagem quando eles são colocados em operação. Quando os aços inoxidáveis são aquecidos em uma faixa de temperatura de $500^{\circ} \mathrm{C}$ a $700^{\circ} \mathrm{C}$, o carbono contido no aço reage com o cromo para formar carbonetos de cromo que não possuem as mesmas características da liga de cromo. Essas reações empobrecem o aço e fazem com que ele diminua suas propriedades na zona aquecida. Esta investigação focou-se na determinação da influência da temperatura no ZAT de uma soldagem em aço inoxidável AISI 316, sob processo GTAW, soldada com eletrodo ER316 quando submetida à temperatura de pré-aquecimento antes da soldagem e seu comportamento antes tratamento termico; e determinando que na soldagem uma baixa contribuição de calor deve ser levada em consideração, distribuindo o calor de forma balanceada para que el seja distribuído na peça da maneira mais simétrica possível. Além disso, deve-se assegurar que o nível de fragilização seja o mais baixo possível, tornando a Zona Afetada pelo calor mais estreita.

Palavras-chaves: soldabilidade, aços inoxidáveis austeníticos, pré-aquecimento.

\section{Introducción}

Los aceros inoxidables son aleaciones complejas en las que intervienen un buen número de elementos químicos. Para conseguir una aceptable soldabilidad, el metal aportado y zonas adyacentes deben presentar unas propiedades lo más semejantes posible a las del material base, tanto desde el punto de vista mecánico como de resistencia a la corrosión.

Ciertos elementos como $\mathrm{Cr}$, $\mathrm{Ti}$ y $\mathrm{Nb}$ se combinan ávidamente con elementos intersticiales como C y $\mathrm{N}$, situados entre los átomos metálicos de la malla cristalina. La combinación da lugar a precipitados de carburos, carbonitruros y nitruros, que se localizan preferentemente en los bordes de grano. La precipitación tiene lugar cuando el material inoxidable permanece durante cierto tiempo en la gama de temperatura comprendida entre $500^{\circ} \mathrm{C}$ y $700^{\circ} \mathrm{C}$, a causa de la operación de soldadura.

En la industria de generación de energía para procesos de producción de hidrocarburos se operan aguas residuales desmineralizadas, las cuales se transportan por medio de tubería fabricada en acero inoxidable AISI 316, dado el manejo de partículas oxidantes. Cuando aplicamos soldadura a este tipo de materiales para la construcción de generadores que contienen este tipo de aguas, el acero es sometido a temperaturas variables que pueden generar la formación de carburos de cromo, los cuales afectan la microestructura del material provocando cambios que pueden ser catastróficos en las propiedades de la estructura al ser sometida a procesos de cargas cíclicas durante su vida útil.

El trabajo de investigación propuesto se enfocó en la determinación de la influencia de la temperatura en la zona afectada térmicamente (ZAT) de una soldadura en un acero inoxidable AISI 316, bajo proceso de soldadura GTAW, con un electrodo ER316L. El calor proveniente del proceso de pre y pos soldadura afecta la microestructura del material con la presencia de carburos de cromo, lo que limita la soldabilidad y provoca la disminución en 
las propiedades de este material. Se buscó dar una posible solución a la disipación de estos carburos con la aplicación de tratamientos térmicos. Se realizó una investigación de tipo descriptivo, en el cual se calentaron tres muestras de acero inoxidable a tres diferentes rangos de temperatura y se procedió a soldarlas bajo parámetros exigidos por códigos aplicables.

En este trabajo se determinó y analizó el cambio microestructura de un acero inoxidable AISI 316 sometido a distintos rangos de precalentamiento en un proceso de soldadura GTAW, dando soluciones a la presencia de carburos de cromo por medio de transformación de fases.

Investigaciones previas a este estudio (Almeida y Villena, 2009; Briones et al., 2014; Camacho, 2015) muestran una técnica para aplicar soldadura fuerte capilar de tubería de acero inoxidable austenítico AISI 304. Este tipo de soldadura se produce por calentamiento a temperatura superior a los $450^{\circ} \mathrm{C}$ y temperaturas inferiores al solidus del metal base. El acero inoxidable contiene cromo y sobre los $425^{\circ} \mathrm{C}$ el cromo se desplaza hacia el borde de grano (precipitación de carburos de cromo) y puede iniciar corrosión intergranular.

Como resultado de esta investigación, se aplican algunos métodos de calentamiento como: soplete, resistencia eléctrica, inducción, horno, inmersión e infrarrojo. Se obtuvieron buenos resultados de soldadura con la selección del material de aporte (Asme, 2015).

De la misma forma, en estudios realizados en el área, se ha investigado la microestructura y propiedades mecánicas de una unión disímil hecha de aceros inoxidables AL-6XN y 316L unidos con el proceso de arco de tungsteno con gas (GTAW) utilizando microscopía óptica (MO) y microscopía electrónica de barrido (MEB), ensayo de tensión, análisis de energía dispersiva de rayos $X$ (EDX) y mediciones de micro dureza. Se realizó un análisis del modo de fractura de las probetas de tensión

Los resultados de tensión muestran que los especímenes de la unión soldada presentan mayor resistencia a la tensión y que la fractura ocurre en la ZAT del acero inoxidable 316L. El análisis microestructural muestra la presencia de pequeñas inclusiones de precipitados de titanio en la ZAT del acero 316L.

Como hipótesis se plantea que los calentamientos al material producen una nucleación de carburos que sean de cromo. Además, la aplicación de tratamientos térmicos disipa o disminuye la cantidad de carburos presentes en el material.

\section{Metodología}

El material base se seleccionó por solicitud expresa de la empresa Masa Stork, la cual facilitó el tipo de acero pues este proyecto nació a causa de los diferentes tipos de problemas que se estaban presentando en una planta de manejo de aguas residuales en dicha empresa. Esta trabajaba con un acero inoxidable AISI 316 y después de la soldadura presentaba fallas la tubería por la zona de unión.

Se seleccionaron muestras a partir de tubería de 2 pulgadas de diámetro y $1 / 8$ pulgadas de espesor, guiándonos por la norma ASTM D1587 (ASTM, 2000). se procedió al muestreo captando 13 muestras patrón para el desarrollo de este proyecto, las cuales se dividirían en los siguientes grupos:

- Una muestra patrón (de control) sin ningún tipo de precalentamiento.

- Tres muestras patrón, las cuales se precalentaron a 400,600 y $800^{\circ} \mathrm{C}$.

- Tres muestras patrón, las cuales se precalentaron a 400,600 y $800^{\circ} \mathrm{C}$ y a las que posteriormente se les aplicó un temple.

- Tres muestras patrón, las cuales se precalentaron a 400,600 y $800^{\circ} \mathrm{C}$ y a las que posteriormente se les aplicó un temple-recocido.

- Tres muestras patrón, las cuales se precalentaron a 400,600 y $800^{\circ} \mathrm{C}$ y a las que posteriormente se les aplicó un normalizado.

El principal objetivo del calentamiento es reducir la velocidad de enfriamiento durante la operación de soldadura, con el fin de: modificar la 
microestructura y modificar el nivel y distribución de las tensiones residuales. Desde un punto de vista general, el calentamiento incluye la temperatura entre pasadas cuando se trata de soldadura en multipasadas, es decir, cuando el calor generado durante la soldadura no es suficiente para mantener la temperatura de precalentamiento entre pasadas sucesivas.

Las muestras fueron puestas en muflas de calentamiento, como se observa en la figura 1, calentando 12 probetas de las cuales 4 se calentaron a $400^{\circ} \mathrm{C}$, otras 4 a $600^{\circ} \mathrm{C}$ y las últimas 4 a $800^{\circ} \mathrm{C}$, para luego ser soldadas con las mismas condiciones de soldeo. Esto con el fin de observar la carburación que se presenta en cada rango de temperaturas que se sugieren en este proyecto.

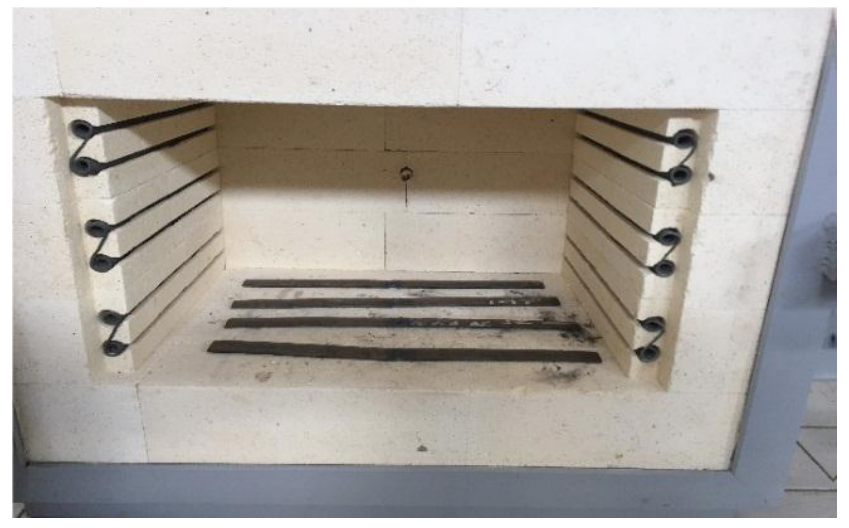

Figura 1. Calentamiento de probetas en muflas. Fuente: elaboración propia de los autores.

Posterior al calentamiento, se realizó metalografía a tres probetas no soldadas que sí tuvieron un calentamiento de la siguiente manera: una probeta de $400^{\circ} \mathrm{C}$, otra de $600^{\circ} \mathrm{C}$ y una final de $800^{\circ} \mathrm{C}$, para verificar la carburación que se presentó en cada nivel de calentamiento. En seguida se ejecutó la soldadura bajo proceso TIG y se continuó con la aplicación de tres tipos de tratamientos térmicos (normalizado, temple y temple-normalizado), los cuales se llevaron a cabo para verificar cuál de estos producía menos carburación en el metal soldado.

Para verificar qué cambios físicos produjo los tratamientos térmicos y soldadura, se verificó por medio de ensayos no destructivos (inspección visual, líquidos penetrantes y radiografía industrial), así como ensayos destructivos, para medir la resistencia y velocidad de deformación de cada probeta.

Así mismo, las muestras soldadas fueron sometidas a ensayos destructivos para verificar la calidad de la soldadura y presencia de discontinuidades o defectos que puedas interferir en el desempeño del material en operación. Luego, se pasaron por metalografía para cuantificar y analizar la presencia de carburos de cromo en la microestructura y su influencia en las características físicas y mecánicas de la ZAT y la unión soldada. Posterior a la soldadura, las muestras fueron sometidas a tres tipos diferentes de tratamientos térmicos en los que se estudió el efecto de estos en la disipación de los carburos y mejora de las propiedades.

En el desarrollo de las etapas de esta investigación, como lo fueron los calentamientos antes del soldeo y la posterior aplicación de tratamientos térmicos, se inspeccionó la calidad del material por diferentes métodos como lo son ensayos no destructivos bajo los métodos de inspección visual, líquidos penetrantes y radiografía industrial. En estas pruebas no se observó defectología tanto interna como externa, por medio de ensayos destructivos tales como metalografía, ensayos de compresión para obtener las características y propiedades de este material y de las soldaduras.

\section{Resultados}

Con el fin de verificar las propiedades que presenta la muestra patrón (de control), se enmarca a continuación la gráfica de desplazamiento que presenta la probeta con relación a una fuerza constante de $500(\mathrm{KN})$. De igual forma, este se trabajó con una velocidad de ensayo de $300 \mathrm{~N} / \mathrm{s}$, para que se presente un offset limite elástico (deformación plástica) del $0.20 \%$.

De la gráfica anterior se puede decir que este material es dúctil ya que tiene una gran 
deformación antes de alcanzar el punto de rotura. Es de anotar que la fractura de esta pieza se produce por el metal base.

De igual manera, se realizó comparación de resultados para los diferentes ensayos destructivos que se muestran en tabla 1, que se explican a través de las figuras $3,4,5$ y 6 .

Se puede evidenciar que la probeta que presenta mayor elasticidad es a la que se le hizo un tratamiento termino por normalizado a $600^{\circ} \mathrm{C}$, presentando con un valor de 3644.8 MPa.
En este caso la probeta con mayor deformación es a la que se le realizó temple con recocido a $600^{\circ} \mathrm{C}$ como tratamiento térmico.

Para este ensayo la probeta con mayor fuerza es a la que se le aplicó tratamiento térmico de temple a $800^{\circ} \mathrm{C}$, característico del proceso térmico, el cual se fortalece y endurece.

En la figura 6 se puede observar que la probeta con mayor punto de rotura es a la que se le realizó temple-recocido a $800^{\circ} \mathrm{C}$, comparada con la probeta patrón.

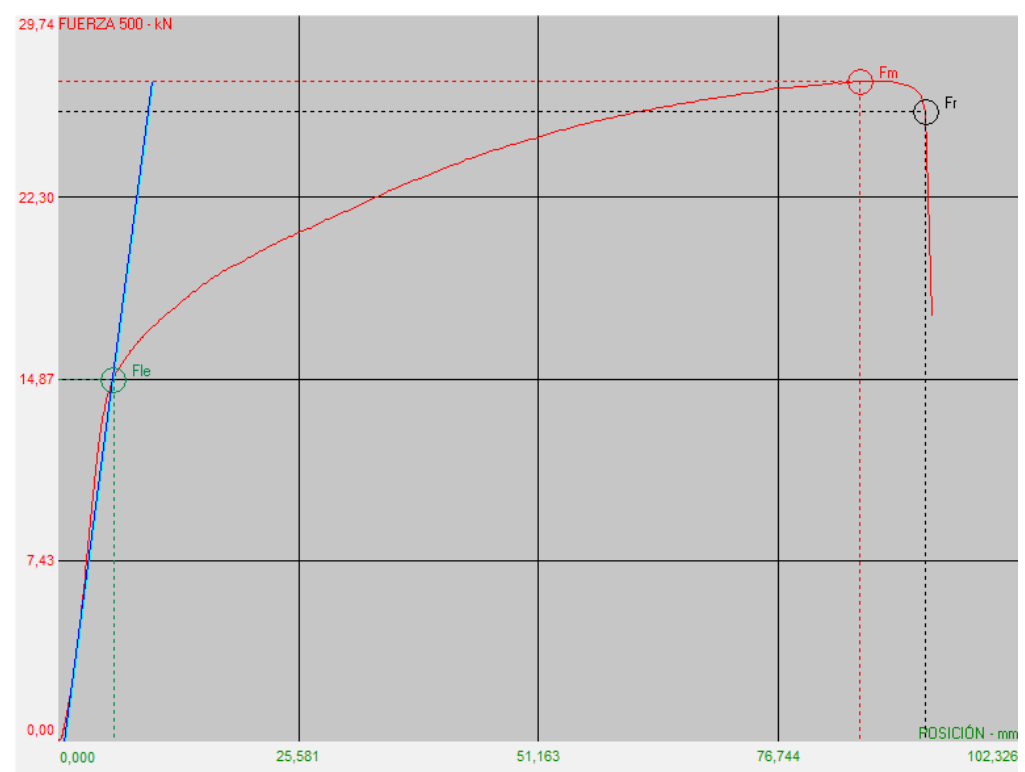

Figura 2. Gráfica fuerza vs. desplazamiento para la muestra patrón.

Fuente: elaboración propia de los autores.

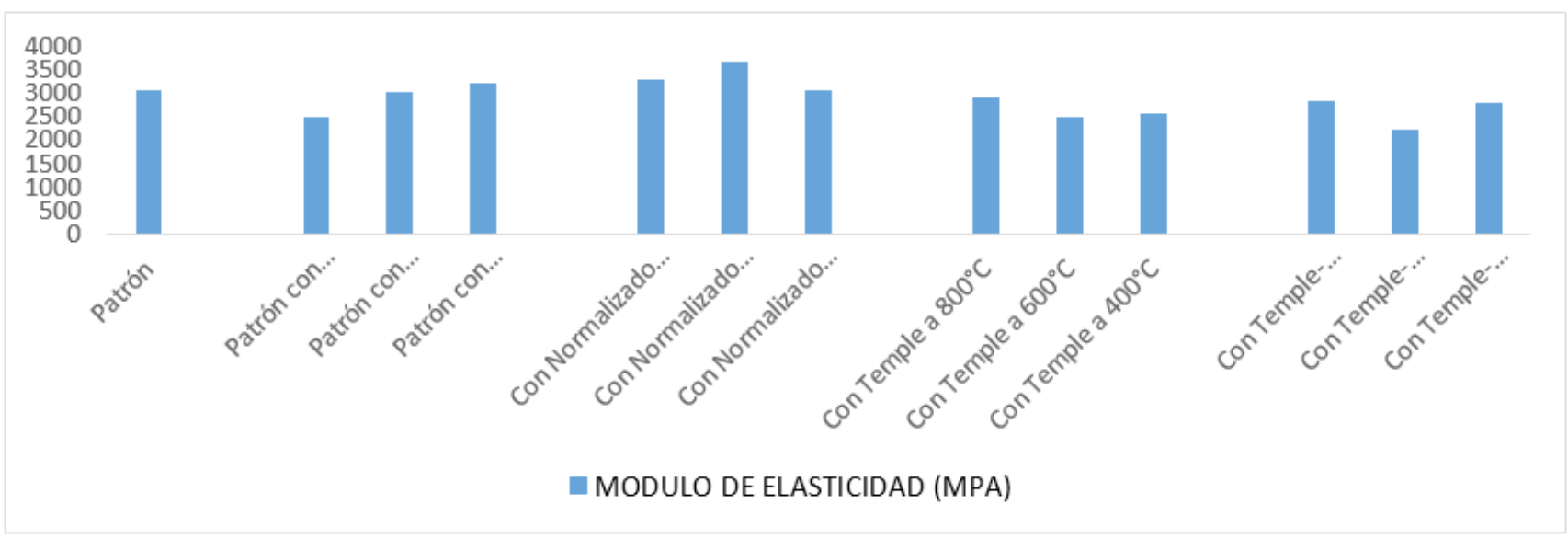

Figura 3. Comparación de resultados del módulo de elasticidad.

Fuente: elaboración propia de los autores. 


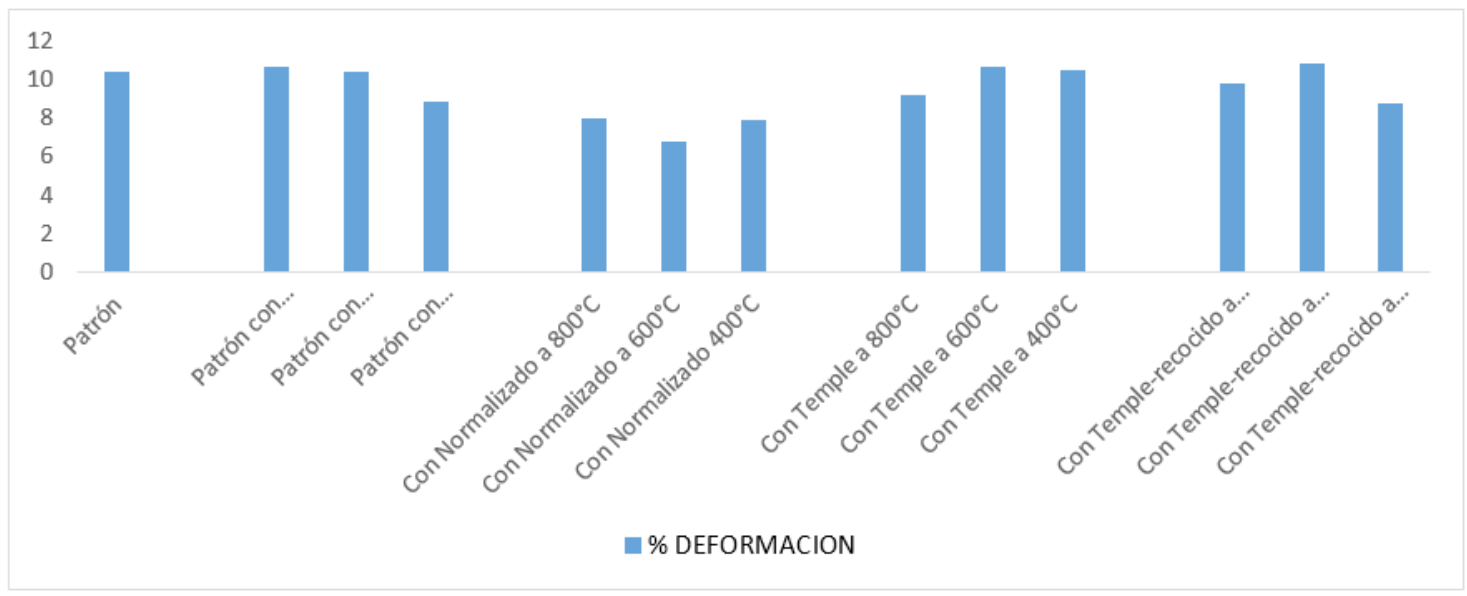

Figura 3. Comparación de resultados del módulo de elasticidad.

Fuente: elaboración propia de los autores.

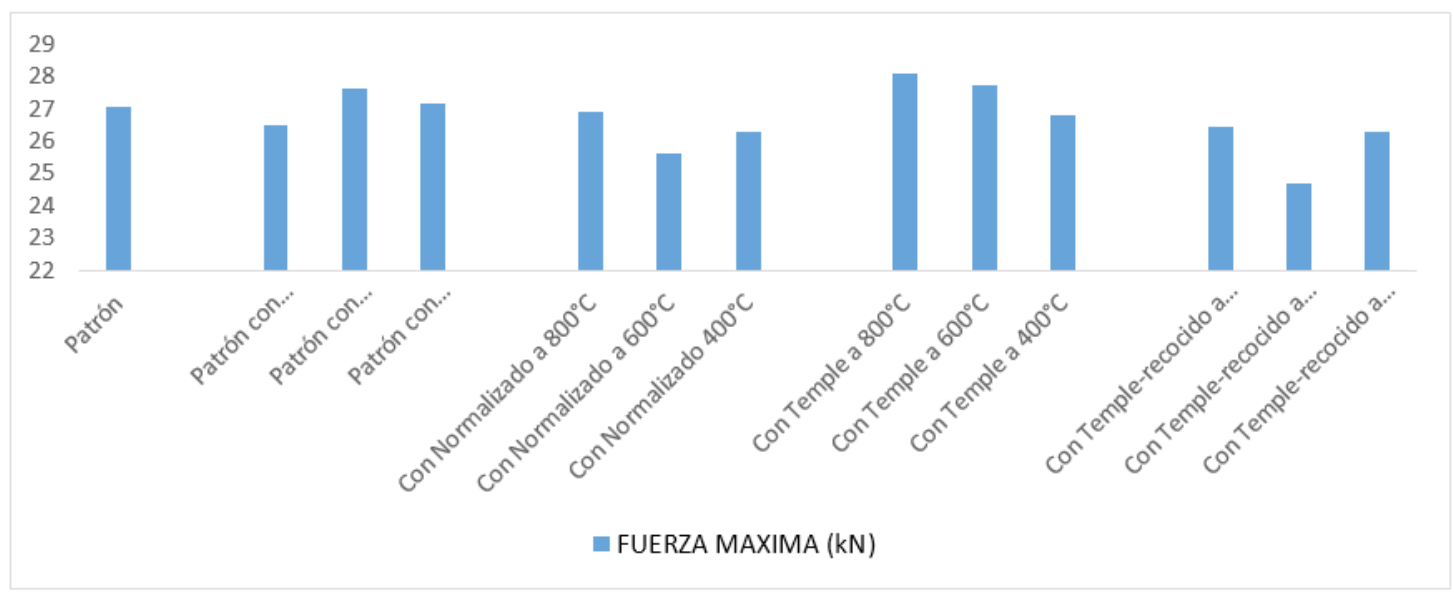

Figura 5. Comparación de resultados de fuerza máxima.

Fuente: elaboración propia de los autores.

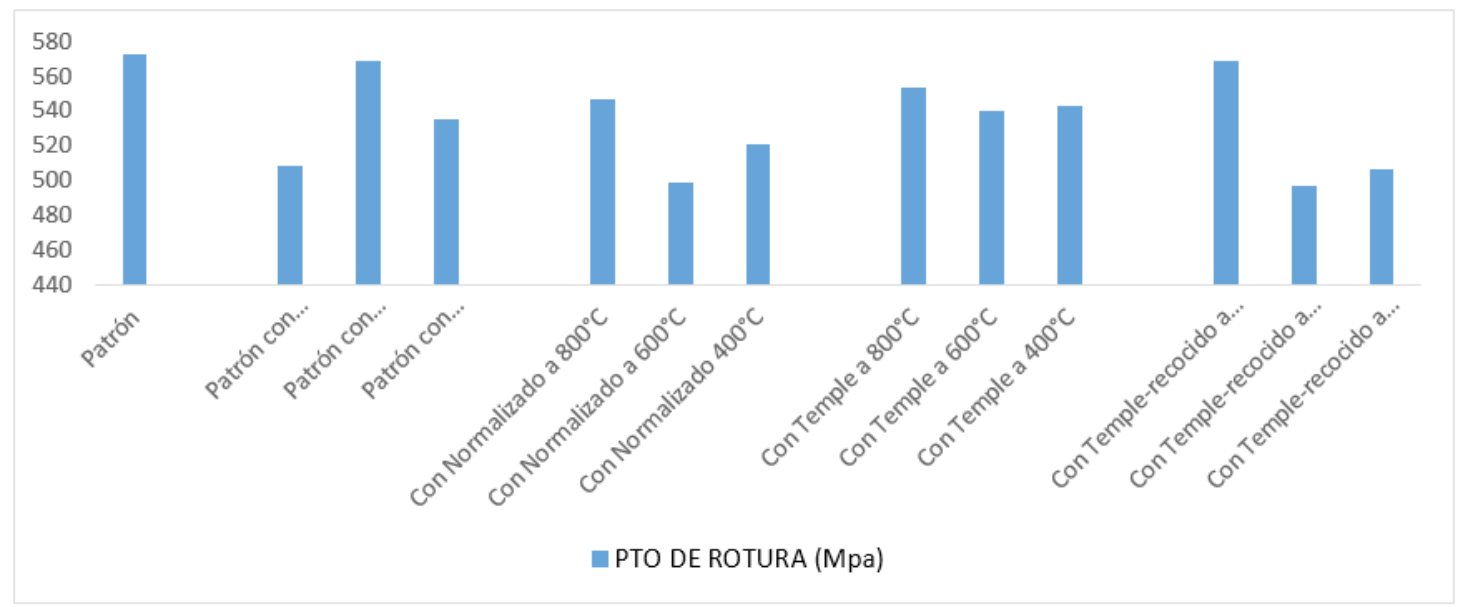

Figura 6. Comparación de resultados de punto de rotura.

Fuente: elaboración propia de los autores. 
Tabla 1. Resultados ensayos de compresión.

\begin{tabular}{|c|c|c|c|c|}
\hline Probetas & $\begin{array}{c}\text { Módulo de } \\
\text { elasticidad (MPA) }\end{array}$ & \% Deformación & Fuerza máxima $(k N)$ & $\begin{array}{c}\text { Punto de } \\
\text { rotura (Mpa) }\end{array}$ \\
\hline Patrón & 3071.973 & 10.41 & 27.032 & 572.104 \\
\hline Patrón con precalentamiento a $800^{\circ} \mathrm{C}$ & 2498.556 & 10.67 & 26.485 & 508.258 \\
\hline Patrón con precalentamiento $600^{\circ} \mathrm{C}$ & 3007.379 & 10.36 & 27.616 & 568.238 \\
\hline Patrón con precalentamiento a $400^{\circ} \mathrm{C}$ & 3193.225 & 8.8 & 27.166 & 535.195 \\
\hline Con normalizado a $800^{\circ} \mathrm{C}$ & 3270.751 & 7.93 & 26.872 & 546.849 \\
\hline Con normalizado a $600^{\circ} \mathrm{C}$ & 3644.818 & 6.74 & 25.607 & 499.153 \\
\hline Con normalizado $400^{\circ} \mathrm{C}$ & 3063.182 & 7.88 & 26.296 & 520.818 \\
\hline Con temple a $800^{\circ} \mathrm{C}$ & 2888.59 & 9.17 & 28.096 & 553.501 \\
\hline Con temple a $600^{\circ} \mathrm{C}$ & 2482.93 & 10.6 & 27.713 & 540.216 \\
\hline Con temple a $400^{\circ} \mathrm{C}$ & 2566.654 & 10.49 & 26.813 & 542.669 \\
\hline Con temple-recocido a $800^{\circ} \mathrm{C}$ & 2831.692 & 9.81 & 26.422 & 568.955 \\
\hline Con temple-recocido a $600^{\circ} \mathrm{C}$ & 2232.706 & 10.83 & 24.66 & 496.387 \\
\hline Con temple-recocido a $400^{\circ} \mathrm{C}$ & 2783.544 & 8.77 & 26.254 & 506.444 \\
\hline
\end{tabular}

Fuente: elaboración propia de los autores.

Tabla 2. Resultados pruebas de dureza.

\begin{tabular}{|c|c|c|c|c|c|}
\hline \multirow{2}{*}{$\begin{array}{c}\text { Probetas } \\
\text { Patrón }\end{array}$} & \multirow{2}{*}{$\begin{array}{l}\text { Material de aporte } \\
\qquad 32\end{array}$} & \multicolumn{2}{|c|}{ Dureza rockwell C } & \multirow{2}{*}{$\begin{array}{c}\text { Final ZAT } \\
25\end{array}$} & \multirow{2}{*}{$\begin{array}{c}\text { Metal base } \\
\quad 32\end{array}$} \\
\hline & & $\begin{array}{l}\text { InICIO LAI } \\
\quad 23.5\end{array}$ & $\begin{array}{l}\text { Centro ZAT } \\
\quad 24.5\end{array}$ & & \\
\hline Patrón con precalentamiento a $800^{\circ} \mathrm{C}$ & 22 & 16 & 22 & 26 & 35 \\
\hline Patrón con precalentamiento $600^{\circ} \mathrm{C}$ & 35 & 15 & 26.5 & 23 & 32 \\
\hline Patrón con precalentamiento a $400^{\circ} \mathrm{C}$ & 23 & 31 & 36 & 27 & 24 \\
\hline Con normalizado a $800^{\circ} \mathrm{C}$ & 28 & 22 & 25 & 23.5 & 36 \\
\hline Con normalizado a $600^{\circ} \mathrm{C}$ & 27.5 & 16 & 16.5 & 31.5 & 35 \\
\hline Con normalizado $400^{\circ} \mathrm{C}$ & 17 & 24 & 18 & 28 & 39.5 \\
\hline Con temple a $800^{\circ} \mathrm{C}$ & 50.5 & 18 & 22 & 27.5 & 40 \\
\hline Con temple a $600^{\circ} \mathrm{C}$ & 47 & 37,5 & 18 & 29 & 51.5 \\
\hline Con temple a $400^{\circ} \mathrm{C}$ & 40 & 12,5 & 18.5 & 31.5 & 38 \\
\hline Con temple-recocido a $800^{\circ} \mathrm{C}$ & 38 & 34 & 32 & 37 & 33 \\
\hline Con temple-recocido a $600^{\circ} \mathrm{C}$ & 27 & 24 & 28.5 & 26 & 24.5 \\
\hline Con temple-recocido a $400^{\circ} \mathrm{C}$ & 28 & 20 & 38.5 & 36 & 32 \\
\hline
\end{tabular}

Fuente: elaboración propia de los autores.

Al comparar los valores obtenidos en este ensayo para las diferentes probetas, con respecto a los de la probeta patrón sin ningún tipo de calentamiento ni tratamiento térmico, se puede concluir que la probeta que fue normalizada posterior a un calentamiento de $600^{\circ} \mathrm{C}$ posee muy buenas propiedades, tanto en tensión de rotura y módulo elástico, pero esta tiene muy bajas características 
en cuanto a \% deformación limite elástico y alargamiento fuerza máxima. Lo anterior quiere decir que esta es muy frágil, viéndose claramente estos resultados en la fractura que sufrió la probeta por la soldadura.

En valores obtenidos para probetas calentadas a $400^{\circ} \mathrm{C}$, tanto sin tratamiento como en normalizado, el temple y el temple-recocido muestran valores muy homogéneos con respecto al patrón. Pero, al observar las micrografías vemos carburación abundante en todas las zonas críticas, dándonos así una defectología que puede afectar el material ya que se empobrece el cromo de este, lo que podría dar paso a problemas como fragilización por corrosión.
Los perfiles de dureza para estas probetas arrojaron resultados acordes al tipo de material y a los tratamientos térmicos que se aplicaron a estos, encontrando de esta manera unas durezas similares a la del acero sin ningún tipo de calentamiento ni tratamiento térmico en las probetas normalizadas y en las que fueran calentadas. Del mismo modo, se ve un aumento gradual en las que fueron templadas $y$, finalmente, se observa diminución en durezas con respecto a las templadas en el recocido dando un perfil de durezas acorde a los tratamientos aplicados.

Para finalizar, se realizaron pruebas y análisis de metalografías para cada probeta, obteniendo los siguientes resultados, como se aprecia en las figuras 8 a 11.

\section{DUREZA ROCKWELL C}

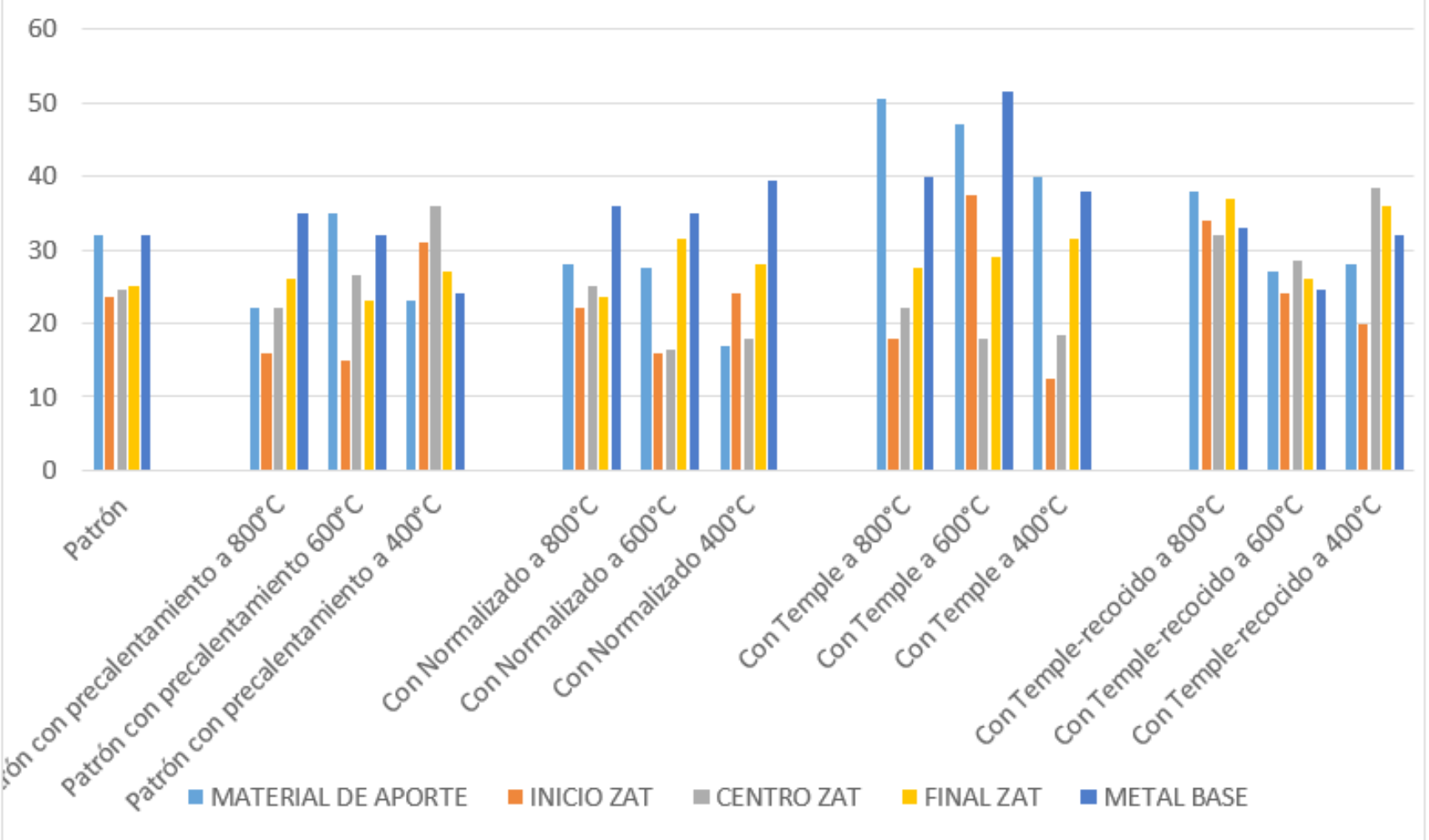

Figura 7. Comparación de dureza.

Fuente: elaboración propia de los autores. 


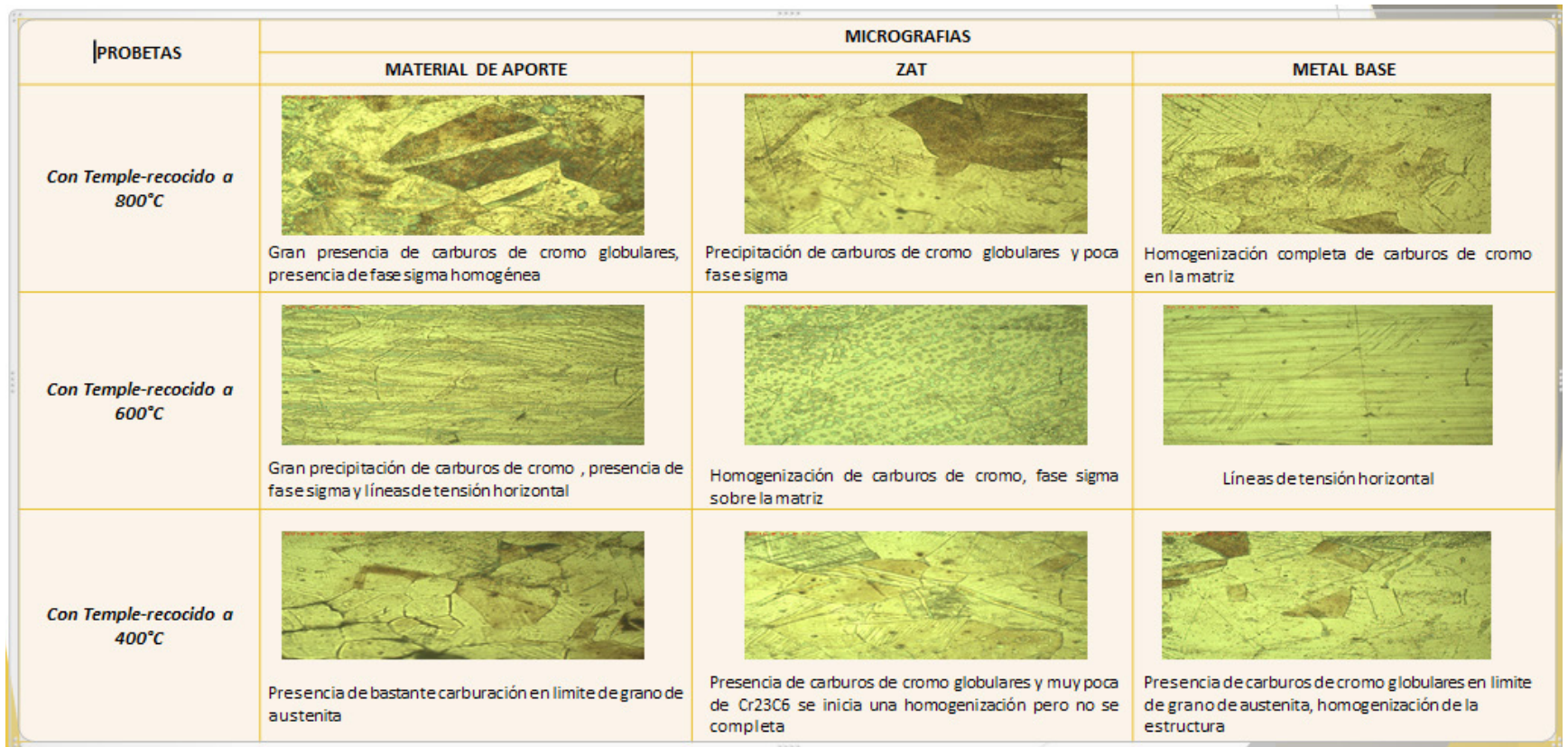

Figura 8. Analisis de micrografias.

Fuente: elaboración propia de los autores.

\begin{tabular}{|c|c|c|c|}
\hline \multirow{2}{*}{ PROBETAS } & \multicolumn{3}{|c|}{ MICROGRAFIAS a 500X } \\
\hline & MATERIAL DE APORTE & ZAT & METAL BASE \\
\hline Con Normalizado & $\begin{array}{l}\text { Precipitación de carburos de cromo en } \\
\text { limites de grano, líneas de tensión } \\
\text { horizontal. }\end{array}$ & $\begin{array}{l}\text { Precipitación de carburos de cromo en matriz } \\
\text { austenitica }\end{array}$ & $\begin{array}{l}\text { Matriz austenitica con leve precipitación de } \\
\text { carburos de cromo, presencia de líneasde } \\
\text { tensión horizontal. }\end{array}$ \\
\hline \multicolumn{4}{|c|}{ Con Normalizado a $600^{\circ} \mathrm{C}$} \\
\hline & $\begin{array}{l}\text { Precipitación de carburos de cromo globulares } \\
\text { dispersos homogéneamente en la matriz, } \\
\text { transgranula e intergranularmente }\end{array}$ & $\begin{array}{l}\text { Mayor preciptación de carburos de cromo globulares en } \\
\text { la zona mas próxima al materialde aporte que al metal } \\
\text { base }\end{array}$ & $\begin{array}{l}\text { Matriz austenitica con mínima precipitación de } \\
\text { carburos de cromo }\end{array}$ \\
\hline \multicolumn{4}{|c|}{ Con Normalizado $400^{\circ} \mathrm{C}$} \\
\hline & $\begin{array}{l}\text { Precipitación de carburos de cromoglobular en } \\
\text { limitesdegrano austenitico }\end{array}$ & $\begin{array}{l}\text { Precipitación de carburos de cromog lobular en limites de } \\
\text { grano austenitico }\end{array}$ & Poca precipitación de carburos de cromo globula \\
\hline
\end{tabular}

Figura 9. Continuación analisis de micrografias.

Fuente: elaboración propia de los autores. 


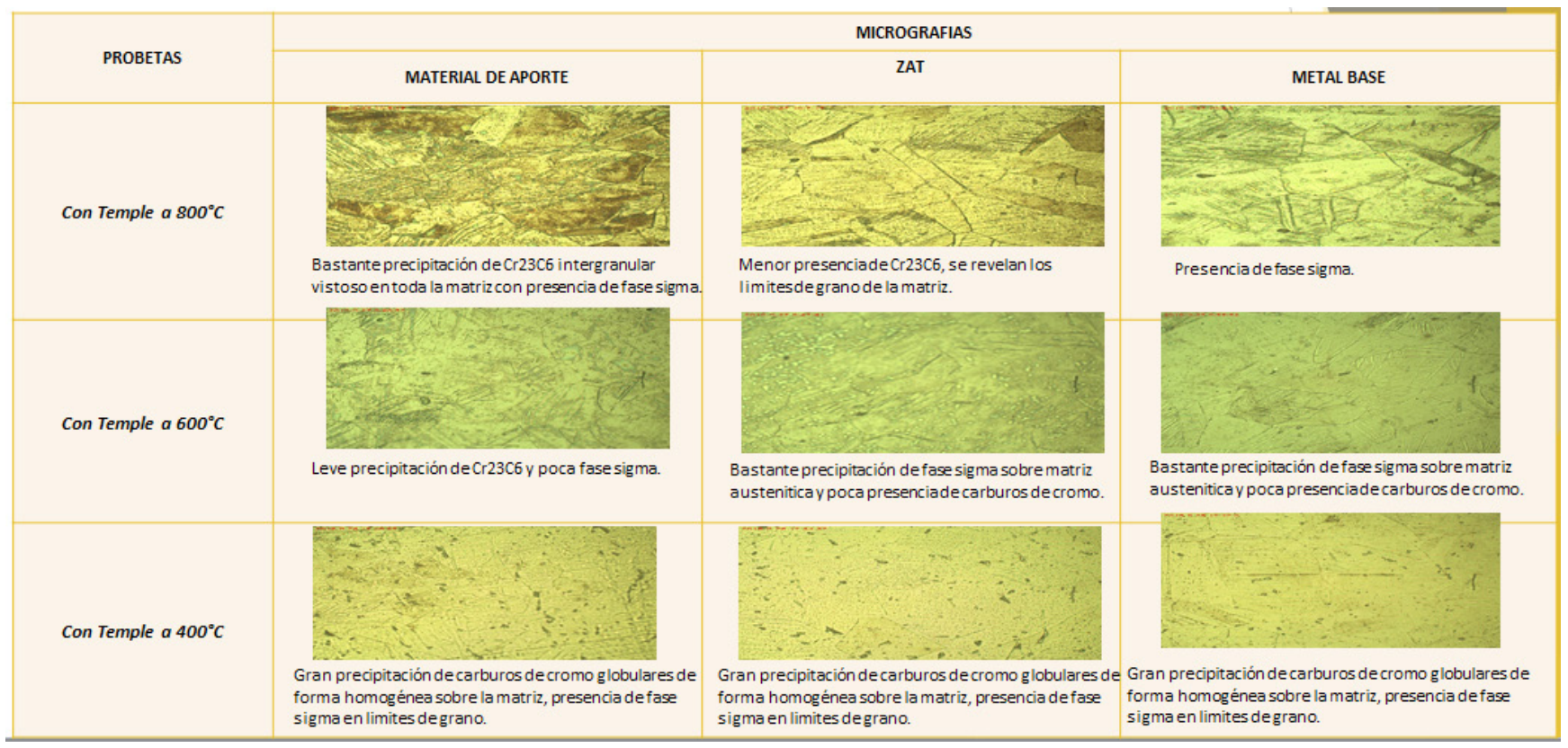

Figura 10. Continuación analisis de micrografias.

Fuente: elaboración propia de los autores.

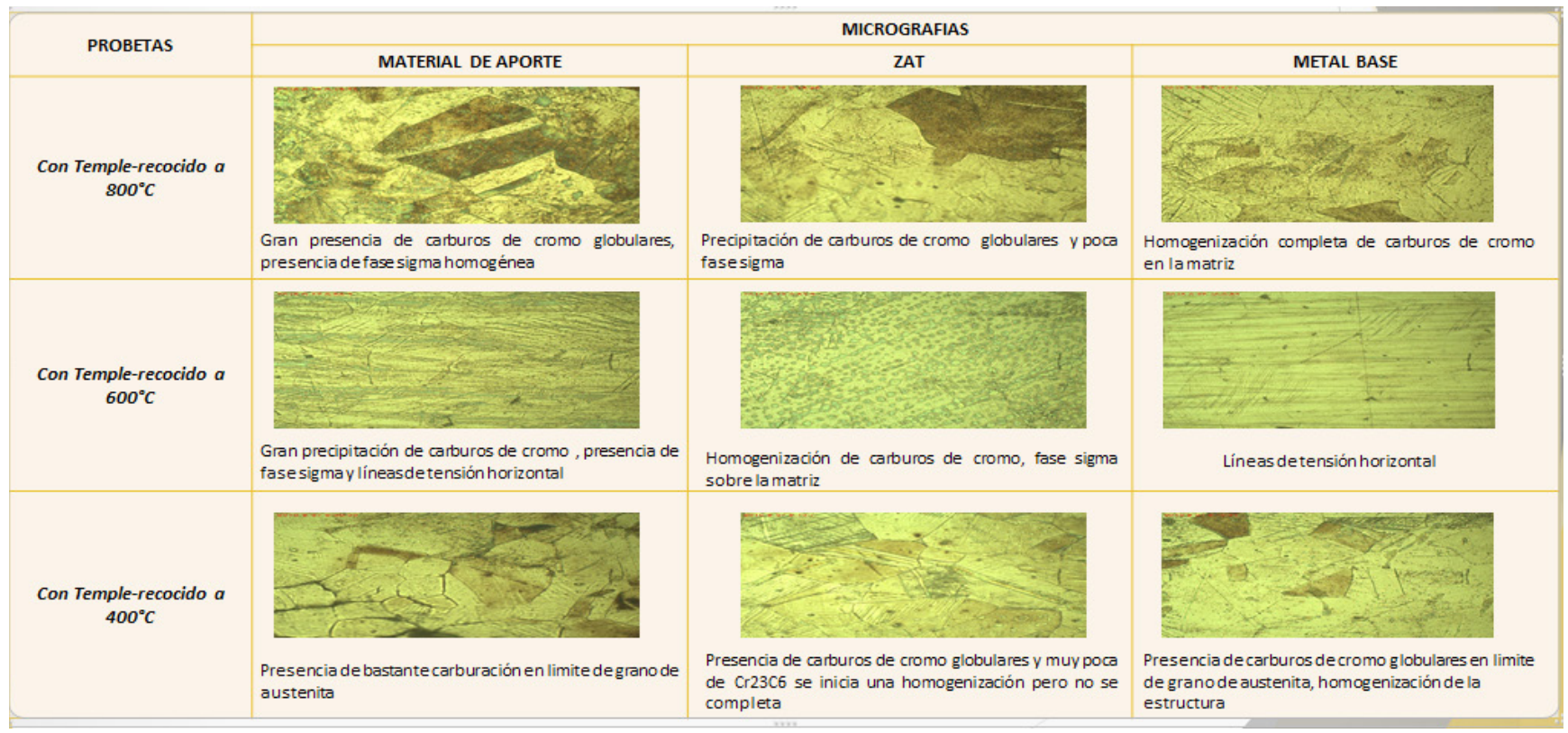

Figura 11. Continuación analisis de micrografias.

Fuente: elaboración propia de los autores. 
Con la aplicación de los distintos tratamientos térmicos (temple, normalizado y temple-recocido) a diferentes temperaturas de precalentamiento $\left(400^{\circ} \mathrm{C}, 600^{\circ} \mathrm{C}\right.$ y $\left.800^{\circ} \mathrm{C}\right)$, se puede decir que las mejores propiedades mecánicas en cuanto a módulo de elasticidad y fuerza máxima de carga se da en el acero AISI 316 soldado con posterior tratamiento térmico de normalizado y precalentado a $600^{\circ} \mathrm{C}$. Lo anterior fue confirmado con el punto de rotura que es el más bajo, lo que demuestra una máxima resistencia mecánica entre la carga máxima y punto de rotura. Sin embargo, se observó que esta probeta falló por la soldadura, obteniéndose un mejoramiento de las propiedades en el metal base y en la ZAT, pero no en la soldadura por formación de carburos de cromo globulares dispersos homogéneamente en la matriz, transgranular e intergranularmente.

\section{Conclusiones}

Durante la aplicación de la soldadura se observó que si esta permanece durante un tiempo prolongado en un intervalo de temperaturas comprendidas entre 500 y $800^{\circ} \mathrm{C}$ hay presencia de precipitación de carburos que influyen en el comportamiento de los componentes en servicio; además, esto se da por la formación de fases intermetálico debido a elementos como cromo y titanio.

Al aplicar tratamientos térmicos posteriores a la soldadura se presenta formación de fase sigma, lo que disminuye la resistencia a la corrosión en la soldadura y en la ZAT, principalmente. Esto incrementa considerablemente la fragilidad por tratarse de una fase bastante dura y como consecuencia también de la ductilidad del material.

Con base en la forma de aplicación de soldadura, para el acero Austenítico AISI 316 estudiado en esta investigación, se recomienda realizar el soldeo teniendo en cuenta una baja aportación de calor, de modo que se distribuya el calor en forma equilibrada para que este sea repartido en la pieza de la forma más simétricamente posible. Además, se debe procurar que el nivel de embridamiento (nivel de nucleación en límites de grano, los cuales producen tensiones residuales) sea lo más bajo posible, así se consigue que la ZAT sea más estrecha.

Se recomienda, con respecto a este estudio, hacer calentamiento para este tipo de acero a temperaturas menores a $400^{\circ} \mathrm{C}$ ya que por encima de esta temperatura se precipitan carburos. De la misma manera se puede decir que es factible un estudio para la soldabilidad de este acero con calentamientos antes de la aplicación del soldeo en un rango de $200^{\circ} \mathrm{C}$ a $400^{\circ} \mathrm{C}$.

\section{Referencias}

Almeida Maldonado, S. L. y Villena Carrasco, C. L. (2009). Soldadura fuerte de tubería de acero inoxidable austenítico (tesis de grado). Escuela Politécnica Nacional, Quito-Ecuador. Recuperado de http://bibdigital.epn.edu.ec/ bitstream/15000/1615/1/CD-2309.pdf.

American Society of Mechanical Engineers - Asme (2015). Boiler and pressure vessel, section IX Qualification standard for welding and brazing procedure, welders, brazers, and welding and brazing operator. Nueva York, EE. UU.

ASTM D1587 (2000). Standard practice for thin-walledtube sampling of soils for geotechnical purposes. West Conshohocken, EE. UU.: ASTM International.

Briones, R., Ruíz, A., Rubio C. y Carreon H. (2014). Caracterización microestructural y mecánica de una soldadura disímil de aceros inoxidables 316L/AL-6XN. Rev. LatinAm. Metal. Mat., 34(2), 306-315.

Camacho, B. A. (2015). Evaluación de la resistencia a la corrosión de uniones soldadas de acero inoxidable mediante proceso GTAW y material de aporte tubular (tesis de grado). Pontificia Universidad Católica del Perú, Perú. Recuperado de http://tesis.pucp.edu.pe/repositorio/ handle/123456789/6073.

Dainoriz, G., Eglimar, R., Levis, F., Jheyssa, H., José, M., Yeison, P. y Osmary, G. (2012). Ensayos no destructivos. Documento de clase, 
Universidad Nacional Experimental Rafael María Baralt, Ojeda, Venezuela.

Gunn, R. (1997). Duplex Stainless Steels. Cambridge, Inglaterra: Woodhead Publishing. DOI: https://doi.org/10.1533/9781845698775

Ibarra, M., Núñez, E. y Huerta, J. M. (2010). Manual de aceros inoxidables. Cerrillos, Chile: $2 \mathrm{M} \mathrm{Im-}$ presores. Recuperado de http://www.indura. cl/Descargar/Manual\%20de\%20Aceros $\% 20$ es?path $=\% 2$ Fcontent $\% 2$ Fstorage $\% 2 \mathrm{Fcl} \% 2 \mathrm{Fbi}-$ blioteca\%2Fd7a1a8fe99fe4b6a9fbed6412df7e93c.pdf.

Lázaro, A. (2012). Propiedades y soldabilidad de los aceros inoxidables. Product Manager Consumibles, European Welding Engineer.
Recuperado de https://www.solysol.com.es/ data/documents/Soldabilidad=20Inox.pdf. Lippold, J. y Kotecki, D (2005). Welding Metallurgy and Weldability of Stainless Steels. EE. UU: John Wiley \& Sons Inc.

Sourmail, T. (2001). Precipitation in creep resistant austenitic stainless steel. Journal Materials Science and Technology, 17(1), 1-14. DOI: https://doi. org/10.1179/026708301101508972

Koseki, T. y Flemings, M. (1996). Solidificaction of undercooled Fe-Cr-Ni alloys part II-microstructural evolution. Metallurgy Materials Transaction, 27(10), 3226-3240. DOI: https://doi. org/10.1007/BF02663873 